\title{
Si-Wu-Tang Alleviates Nonalcoholic Fatty Liver Disease via Blocking TLR4-JNK and Caspase-8-GSDMD Signaling Pathways
}

\author{
Yaxing Zhang $\mathbb{D D}^{1,2}$ Ge Zhou, ${ }^{1,2}$ Zifeng Chen, ${ }^{3,4}$ Weibing Guan, ${ }^{1,2}$ Jiongshan Zhang, \\ Mingmin Bi, ${ }^{5,6}$ Fenglin Wang, ${ }^{1,2}$ Xinchao You, ${ }^{7}$ Yangjing Liao, ${ }^{1,2}$ Shuhui Zheng, ${ }^{8}$ \\ Kangquan $\mathrm{Xu}^{3,4}$ and Hongzhi Yang $\mathbb{D}^{1,2}$ \\ ${ }^{1}$ Department of Traditional Chinese Medicine, The Third Affiliated Hospital, Sun Yat-sen University, Guangzhou, China \\ ${ }^{2}$ Institute of Integrated Traditional Chinese and Western Medicine, Sun Yat-sen University, Guangzhou, China \\ ${ }^{3}$ Biofeedback Laboratory, Xinhua College of Sun Yat-sen University, Guangzhou, China \\ ${ }^{4}$ School of Biomedical Engineering, Xinhua College of Sun Yat-sen University, Guangzhou, China \\ ${ }^{5}$ Department of Otorhinolaryngology, The Seventh Affiliated Hospital, Sun Yat-sen University, Shenzhen, China \\ ${ }^{6}$ Zhongshan School of Medicine, Sun Yat-sen University, Guangzhou, China \\ ${ }^{7}$ Department of Science and Education, \\ The First Affiliated Hospital/School of Clinical Medicine of Guangdong Pharmaceutical University, Guangzhou, China \\ ${ }^{8}$ Research Center for Translational Medicine, The First Affiliated Hospital, Sun Yat-sen University, Guangzhou, China
}

Correspondence should be addressed to Hongzhi Yang; hzyang1960@163.com

Received 30 April 2020; Accepted 2 July 2020; Published 11 August 2020

Academic Editor: Srinivas Nammi

Copyright (C) 2020 Yaxing Zhang et al. This is an open access article distributed under the Creative Commons Attribution License, which permits unrestricted use, distribution, and reproduction in any medium, provided the original work is properly cited.

\begin{abstract}
Background. Nonalcoholic fatty liver disease (NAFLD) has high global prevalence; however, the treatments of NAFLD are limited due to lack of approved drugs. Methods. Mice were randomly assigned into three groups: Control group, NAFLD group, NAFLD plus Si-Wu-Tang group. A NAFLD mice model was established by feeding with a methionine- and choline-deficient (MCD) diet for four weeks. Si-Wu-Tang was given orally by gastric gavage at the beginning of 3rd week, and it lasted for two weeks. The treatment effects of Si-Wu-Tang were confirmed by examining the change of body weight, serum alanine aminotransferase (ALT) and aspartate transaminase (AST) levels, Oil Red O staining, and hematoxylin and eosin (H\&E) staining of the liver samples and accompanied by steatosis grade scores. The expression and activation of the possible signaling proteins involved in the pathogenesis of NAFLD were determined by western blotting. Results. Mice fed with four weeks of MCD diet displayed elevated serum levels of ALT and AST, while there was decreased body weight. The hepatic Oil Red O staining and H\&E staining showed severe liver steatosis with high steatosis grade scores. All these can be improved by treating with Si-Wu-Tang for two weeks. Mechanistically, the increased hepatic TLR4 expression and its downstream JNK phosphorylation induced by MCD diet were suppressed by Si-Wu-Tang. Moreover, the upregulations of Caspase-8, gasdermin D (GSDMD), and cleaved-GSDMD in liver mediated by MCD diet were all inhibited by Si-Wu-Tang. Conclusions. Treatment with Si-Wu-Tang improves MCD diet-induced NAFLD in part via blocking TLR4-JNK and Caspase-8-GSDMD signaling pathways, suggesting that Si-Wu-Tang has potential for clinical application in treating NAFLD.
\end{abstract}

\section{Background}

Nonalcoholic fatty liver disease (NAFLD) is defined as the presence of $>5 \%$ hepatic steatosis (either by histology or imaging techniques); there must be lack of secondary causes of hepatic fat accumulation, such as significant alcohol consumption, long-term use of a steatogenic medication, or monogenic hereditary disorders [1-3]. NAFLD encompasses a broad spectrum of conditions, from simple steatosis (referred to as nonalcoholic fatty liver (NAFL)), through nonalcoholic steatohepatitis (NASH), to fibrosis, and ultimately cirrhosis and hepatocellular carcinoma (HCC) $[4,5]$. 
Moreover, NAFLD is associated with higher risks of severe extrahepatic diseases, such as cardiovascular diseases [1-3]. The global prevalence of NAFLD is estimated to be approximately $24-25 \%$, and the pooled overall nationwide prevalence of NAFLD is estimated to be $29.2 \%$ in China $[2,6,7]$. Therefore, it is urgent to discover novel strategies for treating NAFLD.

A number of traditional Chinese medicinal formulas provide the promising sources to develop alternative and complimentary medicines for NAFLD therapy and prevention [8-11]. Si-Wu-Tang, a traditional Chinese medicinal formula, including Rehmanniae Radix (Shu Di Huang), Angelica Radix (Dang Gui), Paeoniae Radix (Bai Shao Yao), and Chuanxiong Rhizoma (Chuan Xiong), has been shown to improve the antioxidant level and positively regulate the lipid profile, liver function, and skin integrity and texture in healthy adults [12]. This traditional Chinese medicinal formula was first recorded in Xian Shou Li Shang Xu Duan Mi Fang (《仙授理伤续断秘方》) by Lin Daoren in China Tang Dynasty. Si-Wu-Tang has been traditionally used for treating gynecological diseases, such as relief of menstrual irregularity, dysmenorrhea, uterine bleeding, climacteric syndrome, and other estrogen-related diseases, since it is recorded in the official Chinese medicine classics Tai Ping Hui Min He Ji Ju Fang (《太平惠民和剂局方》) in China Song Dynasty [13-20].

By the basic theory of Traditional Chinese Medicine, SiWu-Tang is a blood-building decoction (Chinese Medical Concept: Bu-Xue/补血) to improve a deficiency of blood (血 虚) [13, 21-23]. The animal studies indicated that Si-WuTang has strong abilities to improve blood deficiency induced by radiation [21, 24, 25], or cyclosphosphamide [26], or the compound methods of bleeding, starved feeding, and exhausting [27]. Mechanistically, Si-Wu-Tang improved hematopoietic function of bone marrow by modulating apoptosis, proliferation, and differentiation-related genes expression in haematopoietic stem/progenitor cell [26], alleviated disorders of carbohydrate and lipid metabolisms, disorders of immune function, and the damage of mitochondria and lymphocyte observed in blood deficiency animal models [21, 28, 29]. Si-Wu-Tang administration before irradiation reduced the frequency of radiation-induced apoptosis in crypt of intestine [22] and alleviated intestinal inflammatory processes and protected against intestinal mucosa injury [25]. However, its effects on NAFLD are not clear.

The changes in diet, gut microbiome and the sedentary lifestyle-associated behavior, and the genetic or epigenetic backgrounds that determine relative susceptibility to NAFLD, lead to increased metabolic substrate delivery to the liver and activation of systemic inflammatory changes, causing insulin resistance $[30,31]$. These changes drive increased circulating inflammatory cytokines to induce hepatocellular oxidative stress or endoplasmic reticulum stress and modify cell-cell crosstalk, resulting in cell injury or death, inflammation, fibrogenesis and genomic instability that predispose to cirrhosis and $\operatorname{HCC}[4,30,31]$. Therefore, inflammation, insulin resistance, oxidative stress, and cell death are implicated in the pathogenesis of NAFLD. Among the above pathogenesis, TLR4-mediated innate immune signaling plays an essential role in the development of NAFLD. TLR4 is involved in the pathogenesis of fructoseinduced, high-fat and high-cholesterol diet-induced, or methionine- and choline-deficient (MCD) diet-induced hepatic steatosis in mice [32-35]. Recent studies indicated that liver localization of lipopolysaccharides (LPS), which is the ligand of TLR4, is increased in both human and the experimental NAFLD mice model [36]. Mechanistically, TLR4-induced JNK phosphorylation and their downstream Caspase- 8 activation contribute to the pathogenesis of NAFLD [37-49]. Therefore, hepatocyte-specific Caspase-8 knockout ameliorates the development of MCD diet-induced NASH by modulating liver injury and attenuates alcoholic hepatic steatosis in mice [39, 50]. Moreover, Caspase- 8 activation can lead to the cleavage of gasdermin D (GSDMD) [51-53]. Cleaved-GSDMD forms membrane pores that lead to cytokine release and/or programmed lytic cell death, called pyroptosis [54]. GSDMD plays a key role as a pyroptosis executor in the pathogenesis of NASH by regulating lipogenesis, promoting proinflammatory cytokines secretion, exacerbating NF-кB activation, thus directly or indirectly facilitating liver fibrosis and lipogenesis [55]. Therefore, these innate immune signaling pathways are the essential drug targets for treating NAFLD.

Si-Wu-Tang has strong anti-inflammatory and antioxidative effects $[12,13,17,56-60]$; it can improve carbohydrate and lipid metabolisms in blood deficiency animal models [21, 28, 29] and positively regulate the lipid profile and liver function in healthy adults [12]. Based on these, we speculated that $\mathrm{Si}-\mathrm{Wu}$-Tang might have therapeutic effect on NAFLD. If exists, we will further investigate whether the novel inflammatory and cell death mechanisms of NAFLD discussed above are involved in its protective effects.

\section{Methods}

2.1. Preparation of $\mathrm{Si}-\mathrm{Wu}$-Tang. The drug composition of SiWu-Tang (41 g) was according to Tai Ping Hui Min He Ji Ju Fang (the official Chinese medicine classics in China Song Dynasty) and the recent literatures $[14,17,20,26,28$, 29, 61, 62] (Table 1). The four plant materials prepared in ready-to-use forms were bought from DaShenLin Pharmaceutical Group Co., Ltd. (Guangzhou, China). They were identified according to the first volume of the Chinese Pharmacopoeia 2015 edition by Wang Yonggang, an associate professor in Guangdong Engineering and Technology Research Center for Quality and Efficacy Reevaluation of Post-Market Traditional Chinese Medicine, Sun Yat-sen University (Guangzhou, China). The voucher specimens of Shu Di Huang (no. 2019-BL-016), Dang Gui (no. 2019-BL017), Bai Shao Yao (no. 2019-BL-018), and Chuan Xiong (no. 2019-BL-019) were deposited in Biofeedback Laboratory, Xinhua College of Sun Yat-sen University (Guangzhou, China).

The quality standards of the four plant materials of SiWu-Tang conformed to the Chinese Pharmacopoeia 2015 edition. The prescription of Si-Wu-Tang was based on the clinical application method in Department of Traditional 
Table 1: The formula of Si-Wu-Tang (according to Tai Ping Hui Min He Ji Ju Fang (the official Chinese medicine classics in China Song Dynasty) and references [17] and [20]).

\begin{tabular}{|c|c|c|c|c|}
\hline Chinese name & Scientific name (family) & Place of origin & $\begin{array}{c}\text { Harvest } \\
\text { season }\end{array}$ & $\begin{array}{l}\text { Weight } \\
\text { (g) }\end{array}$ \\
\hline $\begin{array}{l}\text { Shu Di Huang (熟地黄, } \\
\text { Rehmanniae) }\end{array}$ & $\begin{array}{c}\text { Root of Rehmannia glutinosa Libosch } \\
\text { (Scrophulariaceae) }\end{array}$ & Henan, China & Autumn & 15 \\
\hline Dang Gui (当归, Angelicae) & Root of Angelica sinensis Diels (Umbelliferae) & Gansu, China & Late Autumn & 10 \\
\hline Bai Shao Yao (白芶药, Paeoniae) & Root of Paeonia lactiflora Pall (Paeoniaceae) & Anhui, China & Summer & 10 \\
\hline Chuan Xiong (川芎, Chuanxiong) & $\begin{array}{c}\text { Rhizome of Ligusticum chuanxiong Hortorum } \\
\text { (Umbelliferae) }\end{array}$ & $\begin{array}{l}\text { Sichuan, } \\
\text { China }\end{array}$ & Summer & 6 \\
\hline
\end{tabular}

Chinese Medicine, The Third Affiliated Hospital, Sun Yatsen University (Guangzhou, Guangdong, China), and the previously described methods [14, 20, 26, 28, 29, 61-63]. Briefly, the dried prescriptions of Si-Wu-Tang $(2 \times, 82 \mathrm{~g})$ were soaked in $200 \mathrm{ml}$ distilled water for 30 minutes. Then, they were decocted twice with boiling water in a pot made of purple clay with an automatic liquid heater ( $\mathrm{HuFu}$ Hardware Factory, Chaozhou, Guangdong, China), and they were condensed to $82 \mathrm{ml}$. Thus, the concentration of Si-Wu-Tang was $1 \mathrm{~g} / \mathrm{ml}$ (dried herb weight/solution), which was filtered and stored at $4^{\circ} \mathrm{C}$ before use $[20,29]$. Si-Wu-Tang solution was prepared every 3 days.

2.2. The Methionine- and Choline-Deficient (MCD) Diet-Induced NAFLD Animal Model and Treatment Protocol. Male C57BL/6J mice were purchased from Guangdong Medical Laboratory Animal Center (Foshan, Guangdong, China). Mice aged about $8-10$ weeks were used in this study. All animals were housed in a temperature-controlled animal facility with a 12-hour light-dark cycle and allowed to obtain rodent chow and water ad libitum. All animals received humane care, and all animal procedures were approved by the Institutional Animal Care and Use Committee of Sun Yat-sen University (no. 2018-057), and these conformed to the Principles of Laboratory Animal Care formulated by the National Institutes of Health guide for the care and use of Laboratory animals (NIH Publications no. 8023, revised 1978) [64].

Mice were randomly assigned to three groups: Control group $(n=10)$, MCD group $(n=10)$, and MCD plus Si-WuTang group $(n=10)$. As previously revealed [65], mice in MCD group and MCD plus Si-Wu-Tang group were ad libitum fed with a methionine- and choline-deficient diet (MCD; MD12052, Mediscience Ltd., Yangzhou, China) for four weeks to induce NAFLD. Mice in Control group were ad libitum fed with an identical diet sufficient in methionine and choline (MCD control; MD12051, Mediscience Ltd., Yangzhou, China) for four weeks. The histologic evidence shown that there was significant hepatic steatosis in mice fed with the MCD diet for 7, 10, or 14 days [66-70]. Therefore, in order to investigate whether $\mathrm{Si}-\mathrm{Wu}$-Tang has the therapeutic effect on MCD diet-induced NAFLD, mice in MCD plus Si$\mathrm{Wu}$-Tang group were daily given $\mathrm{Si}-\mathrm{Wu}$-Tang from the 15 th day at 16:00-17:00 for two weeks. As previously described $[20,28,29,61,62]$, mice received Si-Wu-Tang at the dose of $1 \mathrm{ml}$ per $100 \mathrm{~g}$ body weight ( $1 \mathrm{~g} / 100 \mathrm{~g}$ body weight) orally by gastric gavage (without fasting). On 29th day, the mice were fasted for four hours before sample collection.

2.3. Measurement of Serum ALT and AST Concentrations. Blood samples were collected by cardiac puncture after the mice were euthanized. Samples were allowed to sit for 30 minutes at room temperature for coagulation [71]. Then, they were centrifuged at $3000 \mathrm{rpm}$ at $4^{\circ} \mathrm{C}$ for 10 minutes, and the supernatant serum was collected and stored at $-80^{\circ} \mathrm{C}$ until analysis [71]. The serum levels of alanine aminotransferase (ALT) and aspartate transaminase (AST) were analyzed by an automatic blood chemistry analyzer (HITACHI 7600, Tokyo, Japan) in the Department of Clinical Laboratory, The Third Affiliated Hospital, Sun Yatsen University.

2.4. Histopathology Analysis. After collecting blood samples, livers were harvested for observing histological alterations by hematoxylin and eosin ( $\mathrm{H} \& \mathrm{E})$ staining and Oil Red $\mathrm{O}$ staining as previously revealed $[65,72]$. Briefly, liver sections embedded in paraffin were stained with $\mathrm{H} \& \mathrm{E}$, and Oil Red $\mathrm{O}$ (Sigma, \#00625) staining was performed in frozen liver sections prepared in Tissue-Tek ${ }^{\circledR}$ optimum cutting temperature (O.C.T.) compound (Sakura, \#4583). H\&E sections were graded for hepatic steatosis as previously described $[73,74]$. Briefly, steatosis was scored and the severity was graded, based on the percentage of the total area affected, into the following categories: $0(<5 \%), 1$ (5-33\%), 2 $(>33-66 \%)$, and $3(>66 \%)[73,74]$.

2.5. Western Blotting. The antibody against TLR4 (\#sc293072) was from Santa Cruz Biotechnology (Santa Cruz, $\mathrm{CA}, \mathrm{USA}$ ). The antibodies against c-Jun $\mathrm{NH}_{2}$-terminal kinase (JNK) (\#9252S), p-JNK (\#9255S) and Caspase-8 (\#9746S), anti-rabbit IgG HRP-linked antibody (\#7074S), and anti-mouse IgG HRP-linked antibody (\#7076S) were from Cell Signaling Technology (Danvers, MA, USA). The antibody against GSDMD (\#ab219800) was from Abcam (Cambridge, UK). Glyceraldehyde-3-phosphate dehydrogenase (GAPDH) antibody (\#MB001) was from Bioworld Technology (Qixia District, Nanjing, China).

Western blotting was performed as we have previously described [64]. The proteins were transferred to polyvinylidene fluoride membranes (Millipore, Bedford, MA, USA), which were incubated with primary and 
TABLE 2: The body weight of mice at baseline and after treatment with SWT.

\begin{tabular}{lcc}
\hline Group & Body weight (0 week) & Body weight (4th week) \\
\hline Control & $24.70 \pm 0.82$ & $26.03 \pm 0.89^{* * *}$ \\
MCD & $25.00 \pm 0.82$ & $16.97 \pm 0.62$ \\
MCD + SWT & $24.90 \pm 0.57$ & $18.64 \pm 0.38^{\# \# \#}$ \\
\hline
\end{tabular}

SWT, Si-Wu-Tang; $n=10,{ }^{* * *} p<0.001$ vs. MCD or MCD + SWT; ${ }^{\# \#} p<0.001$ vs. MCD.

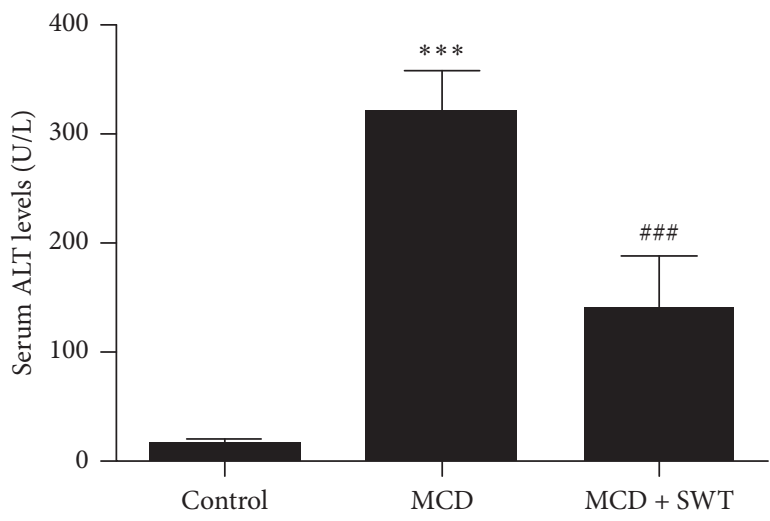

(a)

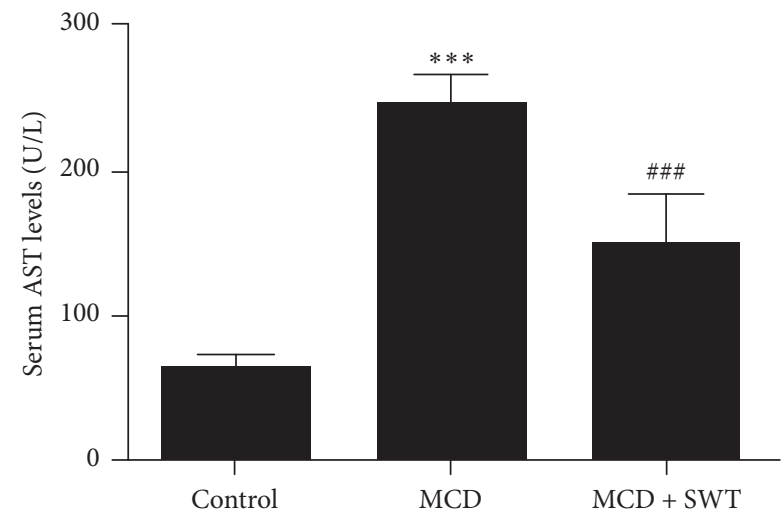

(b)

Figure 1: Si-Wu-Tang decreased MCD diet-induced elevated serum ALT and AST levels. (a) Serum ALT levels in three groups. (b) Serum AST levels in three groups. SWT, Si-Wu-Tang; ALT, alanine aminotransferase; AST, aspartate transaminase; $n=10,{ }^{* * *} p<0.001$ vs. Control; ${ }^{\# \# \# ~} p<0.001$ vs. Control or MCD.

secondary antibodies by standard techniques. The enhanced chemiluminescence (ChemiDoc XRS + System, Bio-Rad, Hercules, CA, USA) was used to accomplish immunodetection.

2.6. Statistical Analysis. Data were expressed as mean \pm SD. Statistical analyses were performed by one-way analysis of variance (ANOVA) followed by Bonferroni's post-hoc test or by Kruskal-Wallis test followed by Dunn's post-hoc test. A value of $p<0.05$ was considered as significantly different. All statistical analyses were performed using GraphPad Prism 5.0.

\section{Results}

3.1. The Effects of Si-Wu-Tang on Body Weight in MCD DietFed Mice. It has been well known that MCD diet can induce body weight loss in mice [75]. Therefore, we measured the body weight of mice at the beginning and at the end of the experiment. As shown in Table 2, there was no difference in the body weight of mice from these three groups at the beginning. After feeding with four weeks of MCD diet, the body weight decreased significantly in MCD group $(p<0.001$ vs. Control) and in MCD plus Si-Wu-Tang group $(p<0.001$ vs. Control). Moreover, two weeks of Si-Wu-Tang treatment improved the loss of body weight $(p<0.001$ vs. MCD) (Table 2).

3.2. Si-Wu-Tang Improved Liver Damage in Mice Fed with a $M C D$ Diet. In order to investigate whether Si-Wu-Tang has the protective effects on liver damage induced by MCD diet, we determined the serum levels of ALT and AST, which are markers of liver damage. Compared with Control group, mice fed with a MCD diet for four weeks resulted in a major increase in serum $\operatorname{ALT}(319.2 \pm 39.4 \mathrm{U} / \mathrm{L}$ vs. $13.8 \pm 5.7 \mathrm{U} / \mathrm{L}, p<0.001)$ and AST $(247.2 \pm 20.2 \mathrm{U} / \mathrm{L}$ vs. $62.4 \pm 10.3 \mathrm{U} / \mathrm{L}, p<0.001$ ) levels (Figures 1(a) and 1(b)). Two weeks of $\mathrm{Si}-\mathrm{Wu}$-Tang treatment decreased serum ALT $(139.2 \pm 48.6 \mathrm{U} / \mathrm{L}$ vs. $319.2 \pm 39.4 \mathrm{U} / \mathrm{L}, p<0.001)$ and AST $(149.4 \pm 35.3 \mathrm{U} / \mathrm{L}$ vs. $247.2 \pm 20.2 \mathrm{U} / \mathrm{L}, \quad p<0.001)$ levels when compared with MCD group (Figures 1(a) and 1(b)). However, the ALT and AST levels in MCD plus Si$\mathrm{Wu}$-Tang group were still higher than those in Control group $(p<0.001)$ (Figures $1(\mathrm{a})$ and $1(\mathrm{~b}))$. Therefore, the observation that the serum levels of ALT and AST in MCD plus $\mathrm{Si}-\mathrm{Wu}$-Tang group were lower than those in the MCD group indicate a protective effect of $\mathrm{Si}-\mathrm{Wu}$-Tang against liver damage.

3.3. Si-Wu-Tang Alleviated Liver Steatosis in Mice Fed with a MCD Diet. Similar to that in humans with NASH, the characteristic pathology of MCD diet-fed mice revealed macrovesicular lipid accumulation [76, 77]. Oil Red O staining displayed that MCD diet induced severe lipid droplet accumulation in the liver, which was alleviated by SiWu-Tang treatment (Figure 2(a)). Similarly, H\&E staining showed that there were hepatic lipid accumulation as clear macrovacuoles, and the increased steatosis grade scores indicated that MCD diet induced severe liver steatosis; all these were improved by treatment with two weeks of Si-WuTang (Figures 2(b)-2(d)). Thus, Si-Wu-Tang improved liver steatosis in mice fed with a MCD diet. 


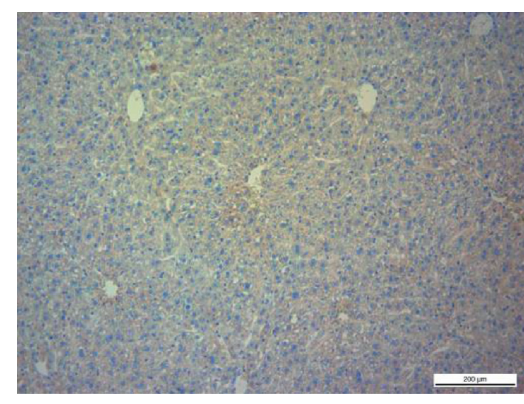

Control

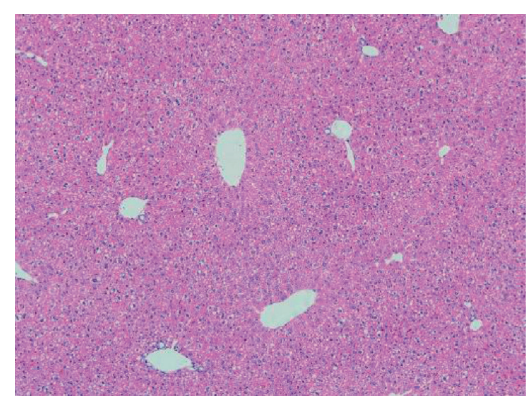

Control

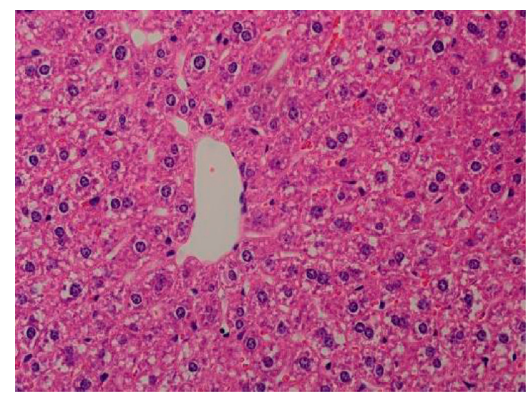

Control

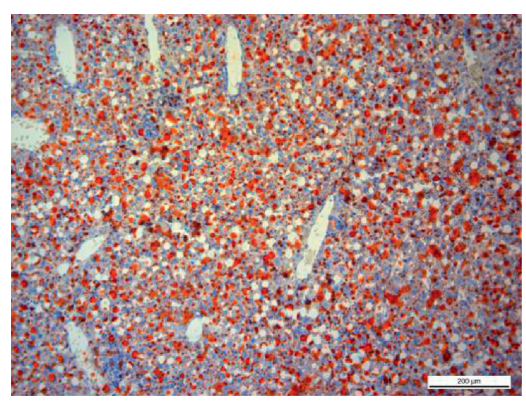

MCD

(a)

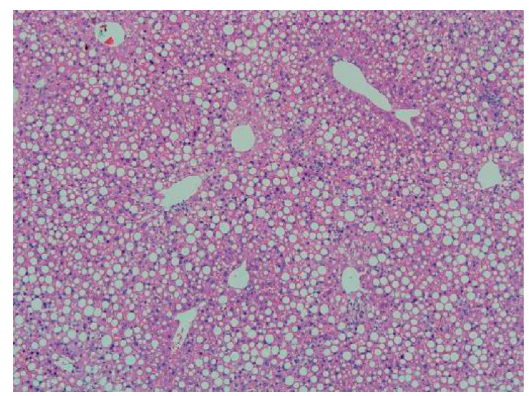

MCD

(b)

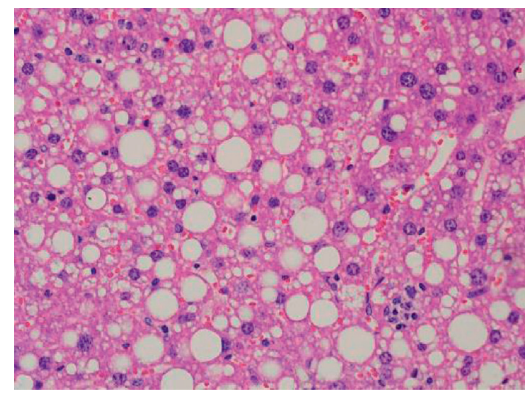

MCD

(c)

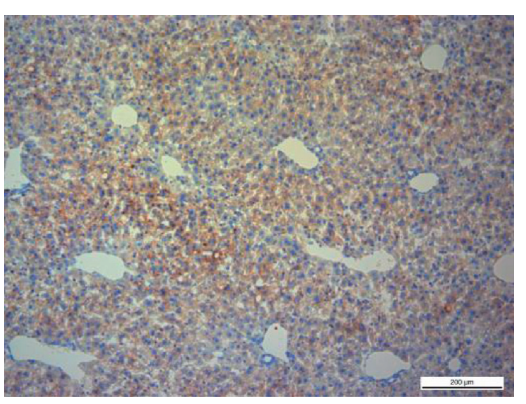

$\mathrm{MCD}+\mathrm{SWT}$

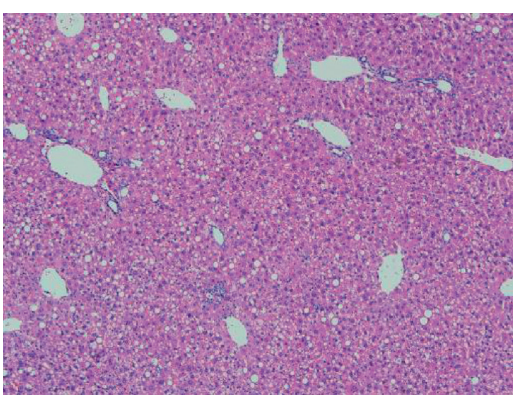

$\mathrm{MCD}+\mathrm{SWT}$

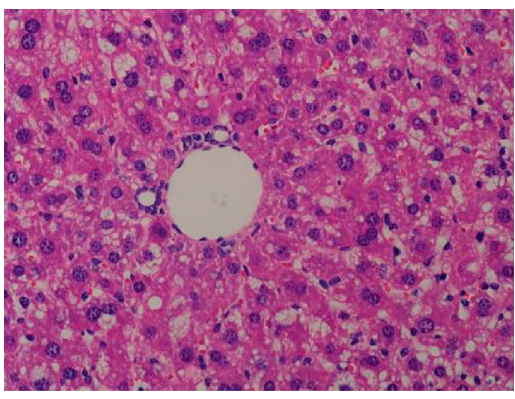

$\mathrm{MCD}+\mathrm{SWT}$

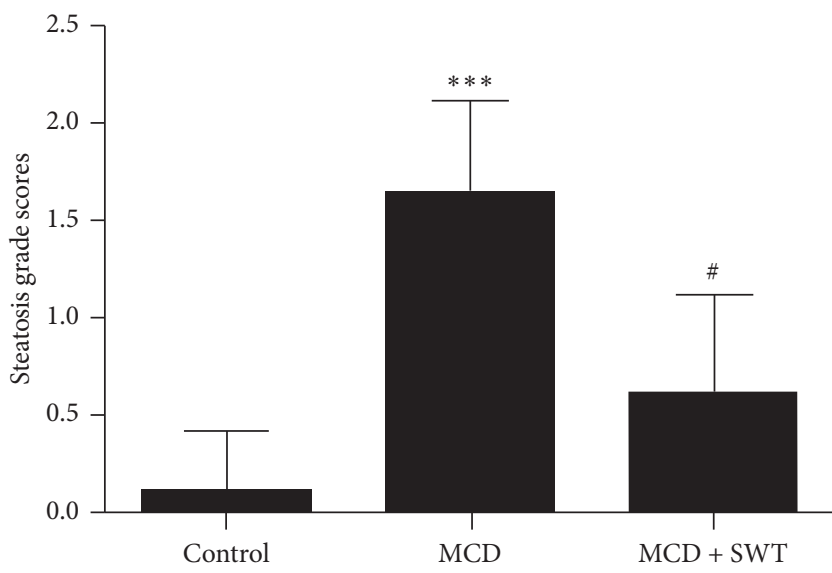

(d)

Figure 2: Si-Wu-Tang improved MCD diet-induced liver steatosis. Liver histology was evaluated by Oil Red O staining (a), H\&E staining ((b) $100^{\times}$; (c) $400^{\times}$), and the steatosis grade scores (d) in three groups. SWT, Si-Wu-Tang; $n=10,{ }^{* * *} p<0.001$ vs. Control; ${ }^{*} p<0.05$ vs. MCD. 
3.4. Si-Wu-Tang Inhibited Hepatic TLR4-JNK Signaling in Mice Fed with a MCD Diet. Immune/inflammatory dysfunctions play important roles in the pathogenesis of NAFLD. Based on the anti-inflammatory effects of Si-WuTang, therefore, we investigated that whether the protective effects of Si-Wu-Tang on NAFLD were related to TLR4 expression. TLR4 was increased in the liver of mice fed with a MCD diet, while it was reduced by two weeks of $\mathrm{Si}-\mathrm{Wu}$ Tang treatment (Figures 3(a) and 3(b)). Subsequently, we examined JNK activation in liver. MCD diet increased hepatic JNK phosphorylation, while JNK activation in liver was inhibited by Si-Wu-Tang (Figures 4(a) and 4(b)).

3.5. Si-Wu-Tang Suppressed Hepatic Caspase-8-GSDMD Signaling in Mice Fed with a MCD Diet. As we have mentioned above, the Caspase-8-GSDMD signaling pathway is essential in the pathogenesis of NAFLD/NASH. Therefore, we investigated whether $\mathrm{Si}-\mathrm{Wu}$-Tang could modulate Caspase-8-GSDMD signaling in liver. As shown in Figure 5, the expressions of hepatic full length Caspase- 8 and the Caspase- 8 active fragment $\mathrm{p} 18$ were increased in mice fed with a MCD diet; Si-Wu-Tang suppressed the expression of full length Caspase- 8 in liver and decreased the Caspase- 8 active fragment p18 levels (with no statistical significance). Moreover, the expression of GSDMD and cleaved-GSDMD were increased in liver of mice fed with a MCD diet; all these were inhibited by Si-Wu-Tang treatment (Figure 6).

\section{Discussion}

The high global or nationwide prevalence of NAFLD underscores the urgent need for effective and safe therapy. However, there are no medications approved by the U.S. Food and Drug Administration (FDA) or European Medicines Agency for the treatment of NAFLD or NASH [78]. Here, we reported that $\mathrm{Si}-\mathrm{Wu}$-Tang has the therapeutic potential for NAFLD.

The MCD diet-induced NAFLD animal model is very reproducible, in which animals rapidly develop the clinical pathologies from macrovesicular steatosis to hepatic fibrosis [76]. Mechanistically, choline or methionine stimulates the synthesis of phosphatidylcholine and increases the cellular phosphatidylcholine levels, which is required for the secretion of very-low-density lipoprotein (VLDL) and its deficiency induces lipid accumulation in the liver $[76,79]$. The methionine-deficient diet induces mitochondrial S-adenosyl-L-methionine (SAM) and glutathione (GSH) depletion due to perturbing mitochondrial membrane dynamics associated with decreased phosphatidylcholine/ phosphatidylethanolamine ratio $[76,80]$. Moreover, MCD diet impairs mitochondrial $\beta$-oxidation and induces cytochrome P450 2E1 (CYP2E1) expression; ROS produced by CYP2E1 $\omega$-oxidation, coupled with the depletion of hepatic antioxidants (e.g., reduced SAM and GSH), amplify oxidative damage, thus inducing steatohepatitis [81-85]. In this study, we established the MCD diet-induced NAFLD mice model and found that two weeks of Si-Wu-Tang treatment alleviated MCD diet-induced elevated serum ALT and AST

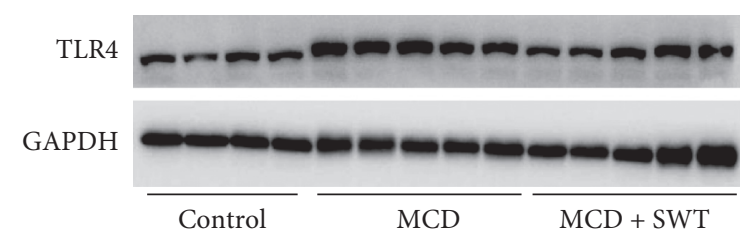

(a)

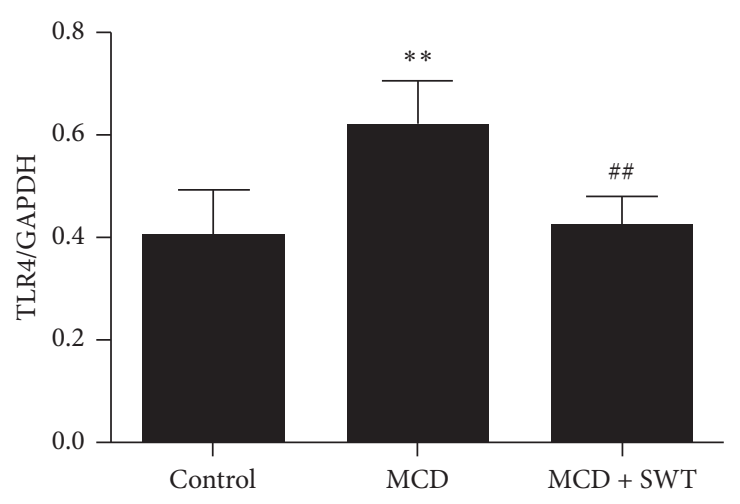

(b)

FIgURE 3: Si-Wu-Tang reduced MCD diet-induced hepatic TLR4 expression. (a) Representative western blot of TLR4 and GAPDH. (b) Quantification of TLR4 expression to GAPDH expression. SWT, Si-Wu-Tang; $n=4-5,{ }^{* *} p<0.01$ vs. Control; ${ }^{\#} p<0.01$ vs. MCD.

levels and improved liver steatosis, supporting the hepatic protective effects of $\mathrm{Si}-\mathrm{Wu}$-Tang.

TLR4 innate immune axis plays an essential role in the pathogenesis of NAFLD, and modulating TLR4 expression and its related signaling pathways have the beneficial effect on NAFLD $[32-36,86,87]$. Genetically, TLR4 deficiency improved NAFLD in mice models [32-34]. Transmembrane BAX inhibitor motif-containing 1 (TMBIM1), which is a multivesicular body (MVB) regulator, protected against NAFLD in mice and monkeys by targeting the lysosomal degradation of TLR4 [87]. In this study, the increased expression of hepatic TLR4 in NAFLD mice was inhibited by Si-Wu-Tang. Besides LPS, the release of high-mobility group box1 (HMGB1) from hepatocytes can also combine with TLR4, thus contributing to the development of NAFLD by inducing JNK activation $[34,37,38]$. Si-Wu-Tang reduced MCD diet-induced hepatic JNK phosphorylation. Therefore, SiWu-Tang alleviated MCD diet-induced NAFLD in part via inhibiting TLR4-JNK signaling.

Stimulation of TLR4 or JNK can also induce Caspase- 8 activation [40-48]. Caspase-8 activation is the characteristic of murine and human alcoholic liver disease (ALD), deficiency of Caspase- 8 in hepatocyte (Casp $\left.8^{\Delta \text { hepatocyte}}\right)$ alleviated steatosis and reduced hepatic triglyceride and free fatty acid (FFA) content in ALD mice model [50]. Moreover, MCD feeding triggered steatosis, hepatic lipid storage, and accumulation of FFA in wild-type livers, which were significantly reduced in Casp $8^{\Delta \text { hepatocyte }}$ animals [39]. Therefore, hepatocyte Caspase- 8 is critical for the pathogenesis of steatohepatitis, drugs targeting Caspase- 8 might be a plausible treatment for NAFLD/NASH [39]. In our study, 


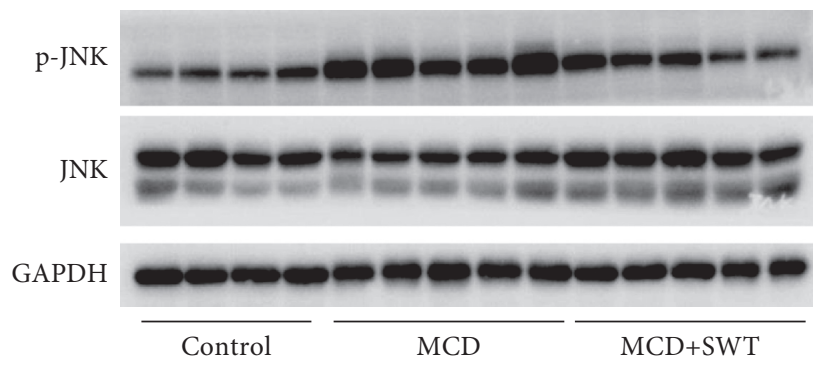

(a)

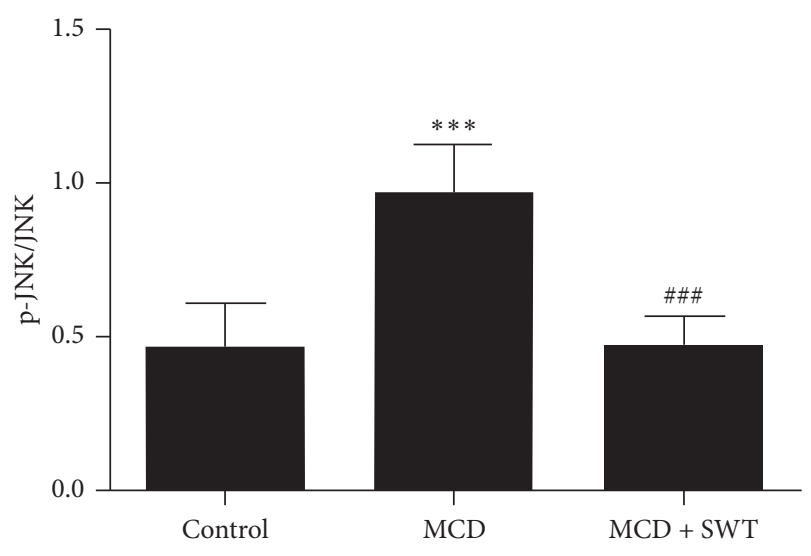

(b)

FIgURE 4: Si-Wu-Tang inhibited MCD diet-mediated JNK activation in liver. (a) Representative western blot of p-JNK, JNK, and GAPDH. (b) Quantification of p-JNK expression to JNK expression. SWT, Si-Wu-Tang; $n=4-5,{ }^{* * *} p<0.001$ vs. Control; ${ }^{\# \# ~} p<0.001$ vs. MCD.

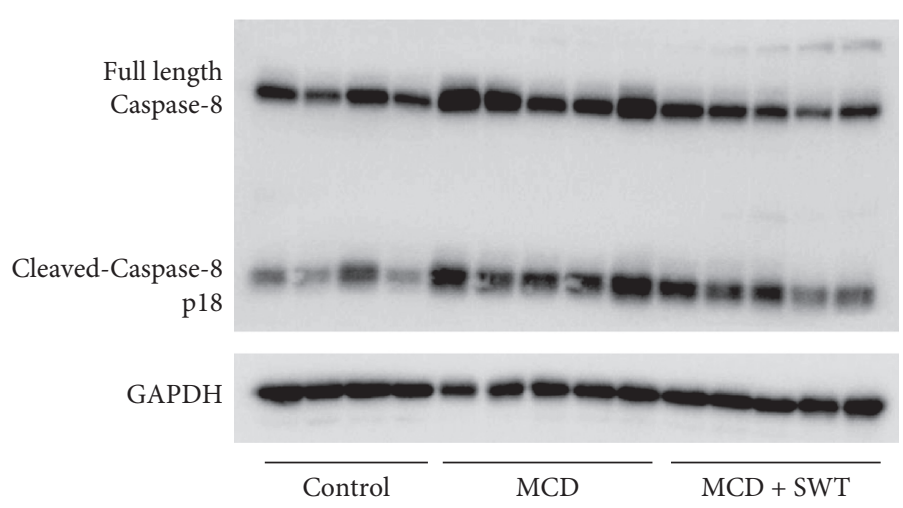

(a)

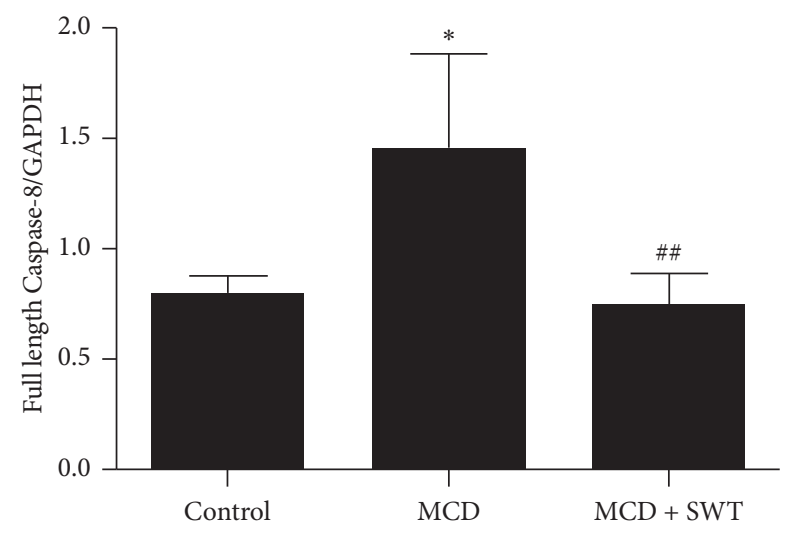

(b)

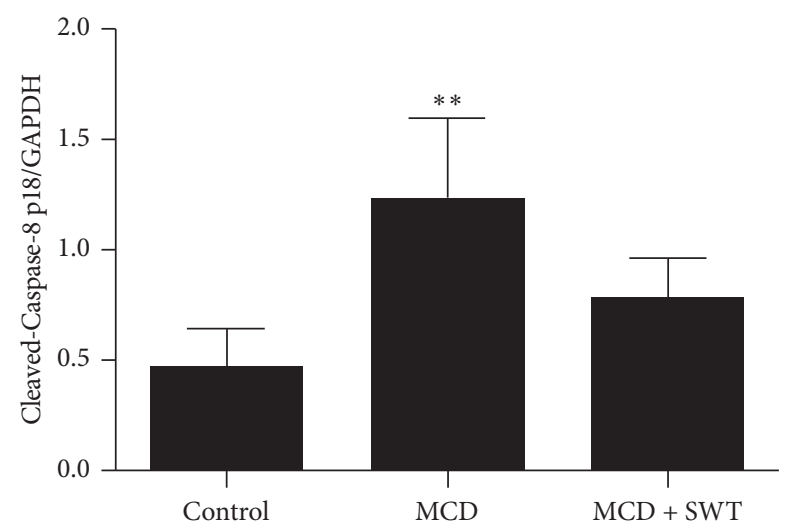

(c)

FIgURE 5: Si-Wu-Tang modulated MCD diet-induced hepatic Caspase-8 expression and activation. (a) Representative western blot of full length Caspase-8, cleaved-Caspase-8 p18, and GAPDH. (b) Quantification of full length Caspase-8 expression to GAPDH expression. (c) Quantification of cleaved-Caspase-8 p18 expression to GAPDH expression. SWT, Si-Wu-Tang; $n=4-5,{ }^{*} p<0.05$ vs. Control; ${ }^{* *} p<0.01$ vs. Control; ${ }^{\#} p<0.01$ vs. MCD.

the MCD diet-triggered hepatic Caspase-8 expression was inhibited by two weeks of $\mathrm{Si}-\mathrm{Wu}$-Tang treatment, which indicated that Caspase-8 is a drug target for $\mathrm{Si}-\mathrm{Wu}$-Tang in treating NAFLD.
Recently, two independent studies indicated that Caspase- 8 activation mediated by TLR 4 resulted in cleavage of GSDMD, leading to pyroptosis [51, 52]. GSDMD and its pyroptosis-inducing fragment GSDMD-N were upregulated 


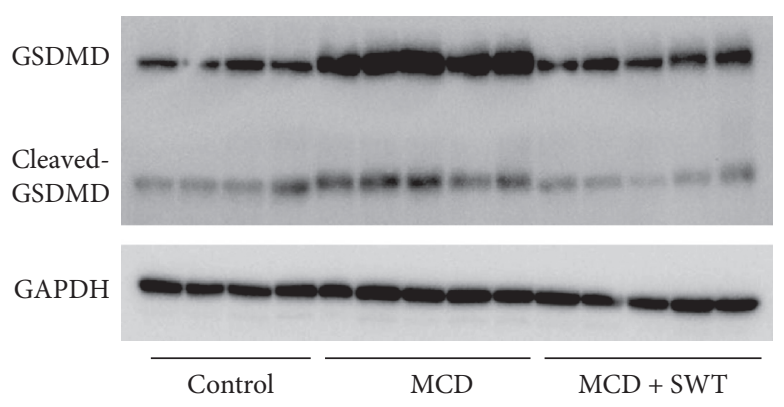

(a)

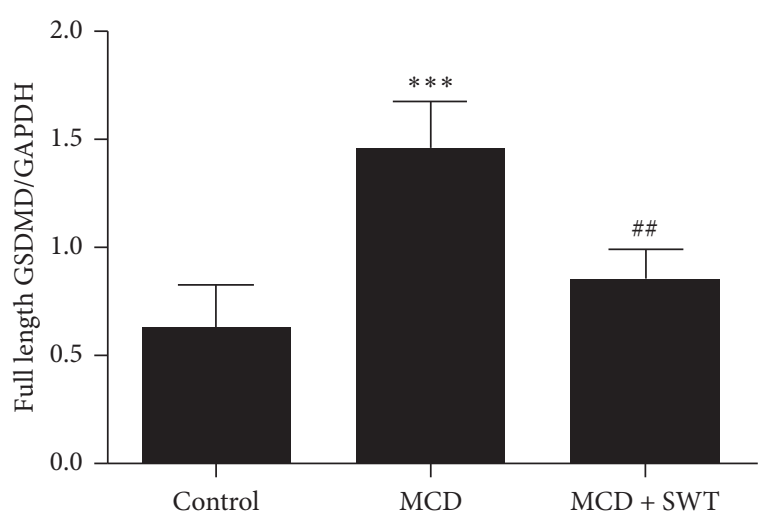

(b)

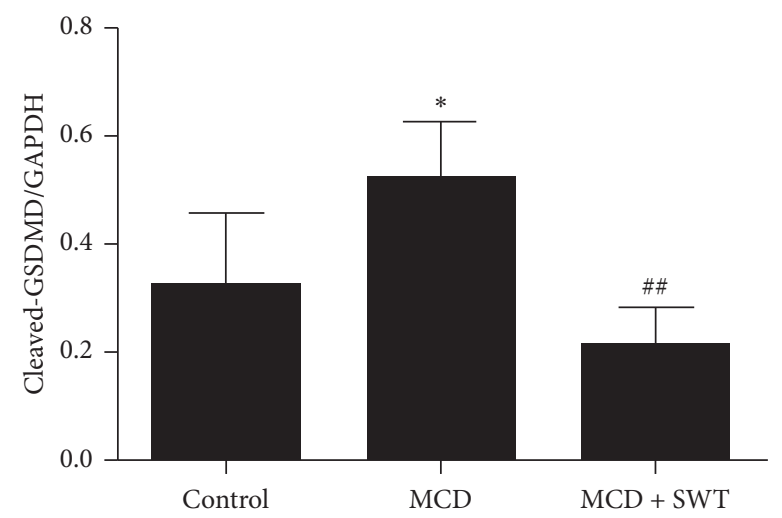

(c)

FIgURE 6: Si-Wu-Tang reduced MCD diet-induced hepatic GSDMD expression and activation. (a) Representative western blot of GSDMD, cleaved-GSDMD, and GAPDH. (b) Quantification of GSDMD expression to GAPDH expression. (c) Quantification of cleaved-GSDMD expression to GAPDH expression. SWT, Si-Wu-Tang; $n=4-5,{ }^{*} p<0.05$ vs. Control; ${ }^{* * *} p<0.001$ vs. Control; ${ }^{\# \#} p<0.01$ vs. MCD.

in liver tissues of human NAFLD/NASH; MCD diet-fed $\mathrm{GSDMD}^{-/-}$mice exhibited decreased severity of steatosis and inflammation compared with wild type littermates [55]. In our study, the expression of hepatic GSDMD and cleavedGSDMD were increased in the NAFLD mice model induced by feeding with a MCD diet. The elevated hepatic GSDMD and cleaved-GSDMD levels were inhibited by Si-Wu-Tang, which indicated that $\mathrm{Si}-\mathrm{Wu}$-Tang can target GSDMD to alleviate NAFLD. Therefore, the protective effect of Si-WuTang on NAFLD was involved in suppressing Caspase-8GSDMD signaling.

Our study confirmed the therapeutic effect of Si-WuTang on MCD diet-induced NAFLD in mice in part via modulating innate immune signaling. However, we only analyzed the TLR4-JNK signaling and Caspase-8-GSDMD signaling pathways; other essential innate immune signaling pathways involved in NAFLD, the expression of inflammatory cytokines, and the key genes involved in hepatic lipids metabolism are not assessed.

\section{Conclusions}

Our present study indicated that $\mathrm{Si}-\mathrm{Wu}$-Tang alleviates MCD diet-induced NAFLD in mice in part via inhibiting
TLR4-JNK signaling and Caspase-8-GSDMD signaling. In conclusion, Si-Wu-Tang may have potential for clinical application in treating NAFLD.

\section{Data Availability}

The data used to support the findings of this study are included within the article.

\section{Conflicts of Interest}

The authors declare that there are no conflicts of interest.

\section{Authors' Contributions}

Zhang Yaxing and Zhou Ge have contributed equally to this work.

\section{Acknowledgments}

This work was supported by Project funded by the China Postdoctoral Science Foundation (Grant number 2019M653238), the Natural Science Foundation of Guangdong Province (Grant numbers 2018A030313657 and 2018A030313733), the National Natural Science Foundation 
of China (Grant numbers 81900376 and 81901447), and the Guangdong Famous Traditional Chinese Medicine Inheritance Studio Construction Project (Grant number 20180137).

\section{References}

[1] Z. Chen, Y. Yu, J. Cai, and H. Li, "Emerging molecular targets for treatment of nonalcoholic fatty liver disease," Trends in Endocrinology \& Metabolism, vol. 30, no. 12, pp. 903-914, 2019.

[2] N. Chalasani, Z. Younossi, J. E. Lavine et al., "The diagnosis and management of nonalcoholic fatty liver disease: practice guidance from the American Association for the Study of Liver Diseases," Hepatology, vol. 67, no. 1, pp. 328-357, 2018.

[3] M. Eslam and J. George, "Genetic contributions to NAFLD: leveraging shared genetics to uncover systems biology," Nature Reviews Gastroenterology \& Hepatology, vol. 17, no. 1, pp. 40-52, 2020.

[4] S. L. Friedman, B. A. Neuschwander-Tetri, M. Rinella, and A. J. Sanyal, "Mechanisms of NAFLD development and therapeutic strategies," Nature Medicine, vol. 24, no. 7, pp. 908-922, 2018.

[5] M. Eslam, L. Valenti, and S. Romeo, "Genetics and epigenetics of NAFLD and NASH: clinical impact," Journal of Hepatology, vol. 68 , no. 2, pp. 268-279, 2018.

[6] F. Zhou, J. Zhou, W. Wang et al., "Unexpected rapid increase in the burden of NAFLD in China from 2008 to 2018: a systematic review and meta-analysis," Hepatology, vol. 70, no. 4, pp. 1119-1133, 2019.

[7] Z. Younossi, Q. M. Anstee, M. Marietti et al., "Global burden of NAFLD and NASH: trends, predictions, risk factors and prevention," Nature Reviews Gastroenterology \& Hepatology, vol. 15, no. 1, pp. 11-20, 2018.

[8] W. F. Hsu, L. Y. Sheen, H. J. Lin, and H. H. Chang, "A review of western and traditional Chinese medical approaches to managing nonalcoholic fatty liver disease," Evidence-Based Complementary and Alternative Medicine, vol. 2016, Article ID 6491420, 12 pages, 2016.

[9] H. Yao, Y.-J. Qiao, Y.-L. Zhao et al., "Herbal medicines and nonalcoholic fatty liver disease," World Journal of Gastroenterology, vol. 22, no. 30, pp. 6890-6905, 2016.

[10] H. Dong, F.-e. Lu, and L. Zhao, "Chinese herbal medicine in the treatment of nonalcoholic fatty liver disease," Chinese Journal of Integrative Medicine, vol. 18, no. 2, pp. 152-160, 2012.

[11] K.-Q. Shi, Y.-C. Fan, W.-Y. Liu, L.-F. Li, Y.-P. Chen, and M.-H. Zheng, "Traditional Chinese medicines benefit to nonalcoholic fatty liver disease: a systematic review and metaanalysis," Molecular Biology Reports, vol. 39, no. 10, pp. 9715-9722, 2012.

[12] H. F. Chiu, Y. H. Wu, Y. C. Shen, S. J. Wang, K. Venkatakrishnan, and C. K. Wang, "Antioxidant and physiological effects of Si-Wu-Tang on skin and liver: a randomized, double-blind, placebo-controlled clinical trial," Chinese Medicine, vol. 11, p. 30, 2016.

[13] M. Liu, R. Ravula, Z. Wang et al., "Traditional Chinese medicinal formula Si-Wu-Tang prevents oxidative damage by activating Nrf2-mediated detoxifying/antioxidant genes," Cell \& Bioscience, vol. 4, no. 1, p. 8, 2014.

[14] Q. D. Liang, C. R. Xiao, Z. C. Ma et al., "Serum fructose concentration in rats after single dose oral administration of Si-Wu-Tang," Drug Discoveries \& Therapeutics, vol. 4, no. 3, pp. 175-178, 2010.
[15] Z.-J. Wang, S.-K. Wo, L. Wang et al., "Simultaneous quantification of active components in the herbs and products of Si-Wu-Tang by high performance liquid chromatographymass spectrometry," Journal of Pharmaceutical and Biomedical Analysis, vol. 50, no. 2, pp. 232-244, 2009.

[16] H. Ohta, J.-W. Ni, K. Matsumoto, H. Watanabe, and M. Shimizu, "Peony and its major constituent, paeoniflorin, improved radial maze performance impaired by scopolamine in rats," Pharmacology Biochemistry and Behavior, vol. 45, no. 3, pp. 719-723, 1993.

[17] G. C. Huang, Y. Z. Tsai, C. J. Lee, H. Y. Chang, and C. C. Wang, "Elucidation of the effects of Si-Wu tang on menstrual disorder patterns through activation of aromatase and antioxidation," Evidence-Based Complementary and Alternative Medicine, vol. 2019, Article ID 4761651, 8 pages, 2019.

[18] B. Zhang, R. Zheng, and Y. Wang, "Study on the antibreast cancer mechanism and bioactive components of Si-Wu-tang by cell type-specific molecular network," Evidence-Based Complementary and Alternative Medicine, vol. 2020, Article ID 2345970, 10 pages, 2020.

[19] P. J. Chang, C. C. Lin, Y. C. Chen et al., "Use of herbal dietary supplement si-Wu-tang and health-related quality of life in postpartum women: a population-based correlational study," Evidence-Based Complementary and Alternative Medicine, vol. 2013, Article ID 790474, 9 pages, 2013.

[20] Q.-D. Liang, Y. Gao, H.-L. Tan et al., "Effects of four Si-WuTang's constituents and their combination on irradiated mice," Biological \& Pharmaceutical Bulletin, vol. 29, no. 7, pp. 1378-1382, 2006.

[21] Z. Ma, C. Huo, S. Zhou et al., "Metabonomic study on siwu tang in radiation-induced blood deficient mice," Zhongguo Zhong Yao Za Zhi, vol. 37, no. 9, pp. 1289-1295, 2012.

[22] S. E. Lee, H. Oh, J. A. Yang et al., "Radioprotective effects of two traditional Chinese medicine prescriptions: Si-Wu-Tang and Si-Jun-Zi-Tang," The American Journal of Chinese Medicine, vol. 27, no. 3-4, pp. 387-396, 1999.

[23] J. Sun, L. Zhang, Y. He et al., "To unveil the molecular mechanisms of qi and blood through systems biology-based investigation into Si-Jun-Zi-Tang and Si-Wu-Tang formulae," Scientific Reports, vol. 6, p. 34328, 2016.

[24] H.-Y. Hsu, Y.-H. Ho, and C.-C. Lin, "Protection of mouse bone marrow by Si-Wu-Tang against whole body irradiation," Journal of Ethnopharmacology, vol. 52, no. 2, pp. 113-117, 1996.

[25] J. Ni, A. L. Romero-Weaver, and A. R. Kennedy, "Potential beneficial effects of Si-Wu-Tang on white blood cell numbers and the gastrointestinal tract of gamma-ray irradiated mice," International Journal of Biomedical Science: IJBS, vol. 10, no. 3, pp. 182-190, 2014.

[26] L. L. Liu, Z. C. Ma, Y. G. Wang et al., "Effects of Siwu decoction on bone marrow protein expression of blood deficiency mice induced by cyclophosphamide," Zhongguo Zhong Yao Za Zhi, vol. 31, no. 14, pp. 1172-1175, 2006.

[27] W. Tan, C. S. Song, H. L. Tan et al., "Hematopoietic effect of siwu decoction in the mice with blood deficiency induced by compound method of bleeding, starved feeding and exhausting," Zhongguo Zhong Yao Za Zhi, vol. 30, no. 12, pp. 926-929, 2005.

[28] C. Huo, Z. Ma, M. Wang, J. Liu, X. Yan, and Y. Gao, "NMRspectroscopy-based metabonomic study on mouse model of blood deficiency syndrome induced by compound method of bleeding, starved feeding and exhausting and effect of Si-WuTang," Zhongguo Zhong Yao Za Zhi, vol. 35, no. 23, pp. 3194-3198, 2010. 
[29] M. Wang, W. Rang, Q. Zhang et al., "NMR-spectroscopybased metabonomic approach to analysis of Si-Wu-Tang, a novel prescription, treated blood deficiency in mice," Zhongguo Zhong Yao Za Zhi, vol. 35, no. 5, pp. 630-634, 2010.

[30] A. J. Sanyal, "Past, present and future perspectives in nonalcoholic fatty liver disease," Nature Reviews Gastroenterology \& Hepatology, vol. 16, no. 6, pp. 377-386, 2019.

[31] Q. M. Anstee, H. L. Reeves, E. Kotsiliti, O. Govaere, and M. Heikenwalder, "From NASH to HCC: current concepts and future challenges," Nature Reviews Gastroenterology \& Hepatology, vol. 16, no. 7, pp. 411-428, 2019.

[32] A. Spruss, G. Kanuri, S. Wagnerberger, S. Haub, S. C. Bischoff, and I. Bergheim, "Toll-like receptor 4 is involved in the development of fructose-induced hepatic steatosis in mice," Hepatology, vol. 50, no. 4, pp. 1094-1104, 2009.

[33] D. Ye, F. Y. L. Li, K. S. L. Lam et al., "Toll-like receptor-4 mediates obesity-induced non-alcoholic steatohepatitis through activation of X-box binding protein-1 in mice," Gut, vol. 61, no. 7, pp. 1058-1067, 2012.

[34] L. Li, L. Chen, L. Hu et al., "Nuclear factor high-mobility group box1 mediating the activation of toll-like receptor 4 signaling in hepatocytes in the early stage of nonalcoholic fatty liver disease in mice," Hepatology, vol. 54, no. 5, pp. 16201630, 2011.

[35] T. Csak, A. Velayudham, I. Hritz et al., "Deficiency in myeloid differentiation factor-2 and toll-like receptor 4 expression attenuates nonalcoholic steatohepatitis and fibrosis in mice," American Journal of Physiology-Gastrointestinal and Liver Physiology, vol. 300, no. 3, pp. G433-G441, 2011.

[36] G. Carpino, M. Del Ben, D. Pastori et al., "Increased liver localization of lipopolysaccharides in human and experimental non-alcoholic fatty liver disease," Hepatology, 2019.

[37] L. T. Gan, D. M. Van Rooyen, M. E. Koina, R. S. McCuskey, N. C. Teoh, and G. C. Farrell, "Hepatocyte free cholesterol lipotoxicity results from JNK1-mediated mitochondrial injury and is HMGB1 and TLR4-dependent," Journal of Hepatology, vol. 61, no. 6, pp. 1376-1384, 2014.

[38] J. M. Schattenberg, R. Singh, Y. Wang et al., "JNK1 but not JNK2 promotes the development of steatohepatitis in mice," Hepatology, vol. 43, no. 1, pp. 163-172, 2006.

[39] M. Hatting, G. Zhao, F. Schumacher et al., "Hepatocyte caspase- 8 is an essential modulator of steatohepatitis in rodents," Hepatology, vol. 57, no. 6, pp. 2189-2201, 2013.

[40] D. Graf, A. K. Kurz, R. Fischer, R. Reinehr, and D. Häussinger, "Taurolithocholic acid-3 sulfate induces CD95 trafficking and apoptosis in a c-Jun N-terminal kinase-dependent manner," Gastroenterology, vol. 122, no. 5, pp. 1411-1427, 2002.

[41] Y. Deng, X. Ren, L. Yang, Y. Lin, and X. Wu, "A JNK-dependent pathway is required for TNF $\alpha$-induced apoptosis," Cell, vol. 115, no. 1, pp. 61-70, 2003.

[42] L. Chang, H. Kamata, G. Solinas et al., "The E3 ubiquitin ligase itch couples JNK activation to TNF $\alpha$-induced cell death by inducing c-FLIPL turnover," Cell, vol. 124, no. 3, pp. 601-613, 2006.

[43] C. Liedtke and C. Trautwein, "The role of JNK2 in toxic liver injury," Journal of Hepatology, vol. 45, no. 5, pp. 762-764, 2006.

[44] Y. Wang, R. Singh, J. H. Lefkowitch, R. M. Rigoli, and M. J. Czaja, "Tumor necrosis factor-induced toxic liver injury results from JNK2-dependent activation of caspase-8 and the mitochondrial death pathway," Journal of Biological Chemistry, vol. 281, no. 22, pp. 15258-15267, 2006.

[45] J. I. Yang, J.-H. Yoon, S. J. Myung et al., "Bile acid-induced TGR5-dependent c-Jun-N terminal kinase activation leads to enhanced caspase 8 activation in hepatocytes," Biochemical and Biophysical Research Communications, vol. 361, no. 1, pp. 156-161, 2007.

[46] J. Maelfait, E. Vercammen, S. Janssens et al., "Stimulation of toll-like receptor 3 and 4 induces interleukin- $1 \beta$ maturation by caspase-8," Journal of Experimental Medicine, vol. 205, no. 9, pp. 1967-1973, 2008.

[47] K. Shenderov, N. Riteau, R. Yip et al., "Cutting edge: endoplasmic reticulum stress licenses macrophages to produce mature IL- $1 \beta$ in response to TLR4 stimulation through a caspase-8- and TRIF-dependent pathway," The Journal of Immunology, vol. 192, no. 5, pp. 2029-2033, 2014.

[48] G. P. Sreekanth, A. Chuncharunee, B. Cheunsuchon, S. Noisakran, P.-t. Yenchitsomanus, and T. Limjindaporn, "JNK1/2 inhibitor reduces dengue virus-induced liver injury," Antiviral Research, vol. 141, pp. 7-18, 2017.

[49] E. Taskin, C. Guven, S. Tunc Kaya et al., "Silencing HMGB1 expression inhibits adriamycin's heart toxicity via TLR4 dependent manner through MAPK signal transduction," Journal of B.U.O.N, vol. 25, no. 1, pp. 554-565, 2020.

[50] F. Hao, F. J. Cubero, P. Ramadori et al., "Inhibition of caspase8 does not protect from alcohol-induced liver apoptosis but alleviates alcoholic hepatic steatosis in mice," Cell Death \& Disease, vol. 8, no. 10, p. e3152, 2017.

[51] J. Sarhan, B. C. Liu, H. I. Muendlein et al., "Caspase-8 induces cleavage of gasdermin D to elicit pyroptosis during yersinia infection," Proceedings of the National Academy of Sciences, vol. 115, no. 46, pp. E10888-E10897, 2018.

[52] P. Orning, D. Weng, K. Starheim et al., "Pathogen blockade of TAK1 triggers caspase-8-dependent cleavage of gasdermin D and cell death," Science, vol. 362, no. 6418, pp. 1064-1069, 2018.

[53] H. I. Muendlein, D. Jetton, W. M. Connolly et al., "cFLIPL protects macrophages from LPS-induced pyroptosis via inhibition of complex II formation," Science, vol. 367, no. 6484, pp. 1379-1384, 2020.

[54] S. Xia, L. R. t. Hollingsworth, and H. Wu, "Mechanism and regulation of gasdermin-mediated cell death," Cold Spring Harb Perspectives in Biology, vol. 12, no. 3, 2020.

[55] B. Xu, M. Jiang, Y. Chu et al., "Gasdermin D plays a key role as a pyroptosis executor of non-alcoholic steatohepatitis in humans and mice," Journal of Hepatology, vol. 68, no. 4, pp. 773-782, 2018.

[56] E. S. Choi, Y. J. Lee, C. S. Seo et al., "Vascular protective role of Samul-Tang in HUVECs: involvement of Nrf2/HO-1 and NO," Evidence-Based Complementary and Alternative Medicine, vol. 2016, Article ID 9580234, 14 pages, 2016.

[57] Z. Wen, Z. Wang, S. Wang et al., "Discovery of molecular mechanisms of traditional Chinese medicinal formula Si-WuTang using gene expression microarray and connectivity map," PLoS One, vol. 6, no. 3, Article ID e18278, 2011.

[58] S. Kojima, K. Inaba, S. Kobayashi, and M. Kimura, "Inhibitory effects of traditional Chinese medicine Shimotsu-to and its included crude fractions on adjuvant-induced chronic inflammation of mice," Biological \& Pharmaceutical Bulletin, vol. 19, no. 1, pp. 47-52, 1996.

[59] E. Tahara, T. Satoh, K. Toriizuka et al., "Effect of Shimotsu-to (a Kampo medicine, Si-Wu-Tang) and its constituents on triphasic skin reaction in passively sensitized mice," Journal of Ethnopharmacology, vol. 68, no. 1-3, pp. 219-228, 1999.

[60] Y. Dai, P. P.-H. But, Y.-P. Chan, H. Matsuda, and M. Kubo, "Antipruritic and antiinflammatory effects of aqueous extract from Si-Wu-Tang," Biological \& Pharmaceutical Bulletin, vol. 25, no. 9, pp. 1175-1178, 2002. 
[61] Z. C. Ma, Y. Gao, H. L. Tang, and S. Q. Wang, "The effects of $\mathrm{Si}-\mathrm{Wu}$-Tang on serum protein of blood deficient mice induced by radiation," Zhongguo Zhong Yao Za Zhi, vol. 28, no. 11, pp. 1050-1053, 2003.

[62] Q. D. Liang, X. Q. Lu, Z. C. Ma et al., "Preliminary study on hematopoietic constituents of Si-Wu-Tang," Zhongguo Zhong Yao Za Zhi, vol. 29, no. 6, pp. 546-549, 2004.

[63] C.-J. Chang, J.-H. Chiu, L.-M. Tseng et al., "Si-Wu-Tang and its constituents promote mammary duct cell proliferation by up-regulation of HER-2 signaling," Menopause, vol. 13, no. 6, pp. 967-976, 2006.

[64] S. Tan, Z. Long, X. Hou et al., "H2 protects against lipopolysaccharide-induced cardiac dysfunction via blocking TLR4-mediated cytokines expression," Frontiers in Pharmacology, vol. 10, p. 865, 2019.

[65] X. Zhang, S. Li, Y. Zhou et al., "Ablation of cytochrome P450 omega-hydroxylase 4A14 gene attenuates hepatic steatosis and fibrosis," Proceedings of the National Academy of Sciences, vol. 114, no. 12, pp. 3181-3185, 2017.

[66] H.-S. Park, B. H. Jeon, S. H. Woo et al., "Time-dependent changes in lipid metabolism in mice with methionine choline deficiency-induced fatty liver disease," Molecules and Cells, vol. 32, no. 6, pp. 571-577, 2011.

[67] M. E. Rinella and R. M. Green, "The methionine-choline deficient dietary model of steatohepatitis does not exhibit insulin resistance," Journal of Hepatology, vol. 40, no. 1, pp. 47-51, 2004.

[68] M. P. Valdecantos, V. Pardo, L. Ruiz et al., "A novel glucagonlike peptide 1 /glucagon receptor dual agonist improves steatohepatitis and liver regeneration in mice," Hepatology, vol. 65, no. 3, pp. 950-968, 2017.

[69] A. S. Henkel, M. S. Elias, and R. M. Green, "Homocysteine supplementation attenuates the unfolded protein response in a murine nutritional model of steatohepatitis," Journal of Biological Chemistry, vol. 284, no. 46, pp. 31807-31816, 2009.

[70] S. W. P. Koppe, A. Sahai, P. Malladi, P. F. Whitington, and R. M. Green, "Pentoxifylline attenuates steatohepatitis induced by the methionine choline deficient diet," Journal of Hepatology, vol. 41, no. 4, pp. 592-598, 2004.

[71] Y. Li, H.-M. Ni, H. Jaeschke, and W.-X. Ding, "Chlorpromazine protects against acetaminophen-induced liver injury in mice by modulating autophagy and c-Jun N-terminal kinase activation," Liver Research, vol. 3, no. 1, pp. 65-74, 2019.

[72] D. Liu, P. Zhang, J. Zhou et al., “TNFAIP3 interacting protein 3 overexpression suppresses nonalcoholic steatohepatitis by blocking TAK1 activation," Cell Metabolism, vol. 31, no. 4, pp. 726-740, 2020.

[73] W. Liang, A. L. Menke, A. Driessen et al., "Establishment of a general NAFLD scoring system for rodent models and comparison to human liver pathology," PLoS One, vol. 9, no. 12, Article ID e115922, 2014.

[74] D. E. Kleiner, E. M. Brunt, M. Van Natta et al., "Design and validation of a histological scoring system for nonalcoholic fatty liver disease," Hepatology, vol. 41, no. 6, pp. 1313-1321, 2005.

[75] S. M. Rahman, J. M. Schroeder-Gloeckler, R. C. Janssen et al., "CCAAT/enhancing binding protein $\beta$ deletion in mice attenuates inflammation, endoplasmic reticulum stress, and lipid accumulation in diet-induced nonalcoholic steatohepatitis," Hepatology, vol. 45, no. 5, pp. 1108-1117, 2007.

[76] S. H. Kim, Y. Lim, J. B. Park et al., "Comparative study of fatty liver induced by methionine and choline-deficiency in
C57BL/6N mice originating from three different sources," Laboratory Animal Research, vol. 33, no. 2, pp. 157-164, 2017.

[77] M. E. Rinella, M. S. Elias, R. R. Smolak, T. Fu, J. Borensztajn, and R. M. Green, "Mechanisms of hepatic steatosis in mice fed a lipogenic methionine choline-deficient diet," Journal of Lipid Research, vol. 49, no. 5, pp. 1068-1076, 2008.

[78] J. Cai, X.-J. Zhang, and H. Li, "Progress and challenges in the prevention and control of nonalcoholic fatty liver disease," Medicinal Research Reviews, vol. 39, no. 1, pp. 328-348, 2019.

[79] Z. M. Yao and D. E. Vance, "The active synthesis of phosphatidylcholine is required for very low density lipoprotein secretion from rat hepatocytes," The Journal of Biological Chemistry, vol. 263, no. 6, pp. 2998-3004, 1988.

[80] F. Caballero, A. Fernández, N. Matías et al., "Specific contribution of methionine and choline in nutritional nonalcoholic steatohepatitis: impact on mitochondrial S-adenosyl-L-methionine and glutathione," Journal of Biological Chemistry, vol. 285, no. 24, pp. 18528-18536, 2010.

[81] G. Serviddio, A. M. Giudetti, F. Bellanti et al., "Oxidation of hepatic carnitine palmitoyl transferase-I (CPT-I) impairs fatty acid beta-oxidation in rats fed a methionine-choline deficient diet," PLoS One, vol. 6, no. 9, Article ID e24084, 2011.

[82] J. Aubert, K. Begriche, L. Knockaert, M. A. Robin, and B. Fromenty, "Increased expression of cytochrome P450 2E1 in nonalcoholic fatty liver disease: mechanisms and pathophysiological role," Clinics and Research in Hepatology and Gastroenterology, vol. 35, no. 10, pp. 630-637, 2011.

[83] Q. M. Anstee and R. D. Goldin, "Mouse models in non-alcoholic fatty liver disease and steatohepatitis research," International Journal of Experimental Pathology, vol. 87, no. 1, pp. 1-16, 2006.

[84] M. D. Weltman, G. C. Farrell, P. Hall, M. Ingelman-Sundberg, and C. Liddle, "Hepatic cytochrome P450 2E1 is increased in patients with nonalcoholic steatohepatitis," Hepatology, vol. 27, no. 1, pp. 128-133, 1998.

[85] M. Weltman, G. Farrell, and C. Liddle, "Increased hepatocyte CYP2E1 expression in a rat nutritional model of hepatic steatosis with inflammation," Gastroenterology, vol. 111, no. 6, pp. 1645-1653, 1996.

[86] U. Vespasiani-Gentilucci, S. Carotti, G. Perrone et al., "Hepatic toll-like receptor 4 expression is associated with portal inflammation and fibrosis in patients with NAFLD," Liver International, vol. 35, no. 2, pp. 569-581, 2015.

[87] G.-N. Zhao, P. Zhang, J. Gong et al., "Tmbim1 is a multivesicular body regulator that protects against non-alcoholic fatty liver disease in mice and monkeys by targeting the lysosomal degradation of Tlr4," Nature Medicine, vol. 23, no. 6, pp. 742-752, 2017. 